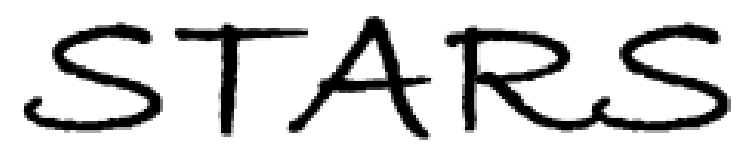

University of Central Florida

STARS

$1-1-2012$

\title{
Remote sensing of neutral temperatures in the Earth's thermosphere using the Lyman-Birge-Hopfield bands of N-2: Comparisons with satellite drag data
}

\author{
Audrey Krywonos \\ University of Central Florida \\ D. J. Murray \\ University of Central Florida \\ R. W. Eastes \\ University of Central Florida
}

\section{A. Aksnes \\ University of Central Florida}

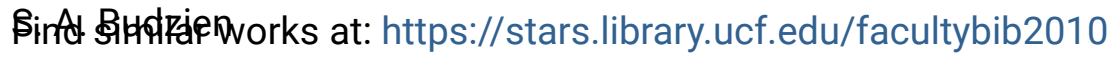

University of Central Florida Libraries http://library.ucf.edu

See next page for additional authors

This Article is brought to you for free and open access by the Faculty Bibliography at STARS. It has been accepted for inclusion in Faculty Bibliography 2010s by an authorized administrator of STARS. For more information, please contact STARS@ucf.edu.

\section{Recommended Citation}

Krywonos, Audrey; Murray, D. J.; Eastes, R. W.; Aksnes, A.; Budzien, S. A.; and Daniell, R. E., "Remote sensing of neutral temperatures in the Earth's thermosphere using the Lyman-Birge-Hopfield bands of N-2: Comparisons with satellite drag data" (2012). Faculty Bibliography 2010s. 2885.

https://stars.library.ucf.edu/facultybib2010/2885

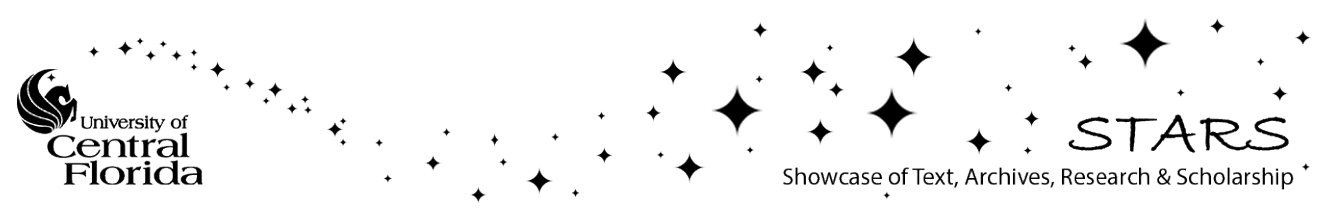




\section{Authors}

Audrey Krywonos, D. J. Murray, R. W. Eastes, A. Aksnes, S. A. Budzien, and R. E. Daniell 


\title{
Remote sensing of neutral temperatures in the Earth's thermosphere using the Lyman-Birge-Hopfield bands of $\mathrm{N}_{\mathbf{2}}$ : Comparisons with satellite drag data
}

\author{
Andrey Krywonos, ${ }^{1}$ D. J. Murray, ${ }^{2,3}$ R. W. Eastes, ${ }^{1,2}$ A. Aksnes, ${ }^{1,4}$ S. A. Budzien, ${ }^{5}$ \\ and R. E. Daniell ${ }^{6}$ \\ Received 10 October 2011; revised 28 June 2012; accepted 9 August 2012; published 18 September 2012.
}

[1] This paper presents remotely sensed neutral temperatures obtained from ultraviolet observations and compares them with temperatures from the NRLMSISE-00 version of the Mass Spectrometer and Incoherent Scatter (MSIS) model (unconstrained and constrained to match the total densities from satellite drag). Latitudinal profiles of the temperatures in the Earth's thermosphere are obtained by inversion of high-resolution $(\sim 1.3 \AA)$

observations of the $(1,1)$ and $(5,4)$ Lyman-Birge-Hopfield $(\mathrm{LBH})$ bands of $\mathrm{N}_{2}$. The spectra are from the High resolution Ionospheric and Thermospheric Spectrograph (HITS) instrument aboard the Advanced Research and Global Observation Satellite (ARGOS). The results indicate that on each day examined there was consistency between the remotely sensed thermospheric temperatures, the densities from coincident satellite drag measurements at adjacent altitudes, and the NRLMSISE-00 model.

Citation: Krywonos, A., D. J. Murray, R. W. Eastes, A. Aksnes, S. A. Budzien, and R. E. Daniell (2012), Remote sensing of neutral temperatures in the Earth's thermosphere using the Lyman-Birge-Hopfield bands of $\mathrm{N}_{2}$ : Comparisons with satellite drag data, J. Geophys. Res., 117, A09311, doi:10.1029/2011JA017226.

\section{Introduction}

[2] Earth's thermosphere is dynamic, responding within hours to changes in both solar irradiance and geomagnetic activity. Understanding the variation of the neutral temperature $\left(T_{n}\right)$ in the thermosphere is a key to understanding the coupling of Earth's upper atmosphere with the space environment. Both the spatial and temporal response, at global scales, of the thermospheric temperatures to solar and geomagnetic changes are essential. While it is of key importance, the availability of temperature data for the lower thermosphere (100-200 km) is currently insufficient for understanding the changes seen in the thermosphere and ionosphere.

[3] Both direct and remote measurements of the thermosphere have been employed, but the available techniques have

\footnotetext{
${ }^{1}$ Florida Space Institute, University of Central Florida, Orlando, Florida, USA.

${ }^{2}$ Department of Physics, University of Central Florida, Orlando, Florida, USA.

${ }^{3}$ Now at National Ground Intelligence Center, Charlottesville, Virginia, USA.

${ }^{4}$ Now at Faculty of Mathematics and Natural Sciences, University of Bergen, Bergen, Norway.

${ }^{5}$ Naval Research Laboratory, Washington, D. C., USA.

${ }^{6}$ Ionospheric Physics, Stoughton, Massachusetts, USA.

Corresponding author: A. Krywonos, Florida Space Institute, University of Central Florida, 12354 Research Pkwy., Partnership I Bldg., Orlando, FL 32826, USA. (krywonos@ucf.edu)

C2012. American Geophysical Union. All Rights Reserved. 0148-0227/12/2011JA017226
}

not provided the spatial and temporal coverage necessary. Due to the low density of the thermosphere, accurate direct measurements are difficult to obtain and are geographically localized. Radar techniques can be used in the daytime, but the instrumentation is large, expensive, and consequently has limited availability. In addition, all ground-based measurements are spatially localized and provide limited coverage over the oceans. While ground-based optical remote sensing is possible, ultraviolet (UV) emissions from the Earth's dayglow, which provide more direct information about the conditions and processes in the upper atmosphere, are absorbed by $\mathrm{O}_{2}$ at altitudes below $100 \mathrm{~km}$ and can only be observed from space.

[4] The Lyman-Birge-Hopfield (LBH) bands, which originate from the $\mathrm{a}^{1} \Pi_{\mathrm{g}}$ state of $\mathrm{N}_{2}$, are one of the emissions most often used for UV remote sensing of the space environment [Christensen et al., 2003; Meier, 1991]. The LBH band emission has primarily been used, along with the OI $135.6 \mathrm{~nm}$ emission, in the determination of the ratio of $\mathrm{O}$ and $\mathrm{N}_{2}$ column densities $\left(\mathrm{O} / \mathrm{N}_{2}\right)$ [Strickland et al., 1995], one of the primary state variables in the thermosphere. However, the emission can also be used to determine rotational temperatures. In one study of the LBH bands of $\mathrm{N}_{2}$ [Aksnes et al., 2006] these rotational temperatures have shown reasonable agreement with neutral temperatures, a second state variable in the thermosphere. In the current study, rotational temperatures in the lower thermosphere-obtained through inversion of $\mathrm{N}_{2}$ LBH $(1,1)$ and $(5,4)$ emissions-are compared with neutral temperatures from the NRLMSISE-00 model [Picone et al., 2002], both with and without constraint 
of the neutral densities using coincident satellite drag measurements.

\section{HITS Instrument and Data}

[5] The LBH emissions used in this study were measured by the High resolution Ionospheric and Thermospheric Spectrograph (HITS) aboard the Advanced Research and Global Observation Satellite (ARGOS) [Dymond et al., 1999]. ARGOS was launched 23 February 1999 into a Sunsynchronous, near-polar, circular orbit $(840 \mathrm{~km})$, with an ascending node crossing time of 14:30 local solar time. The HITS instrument was in operation from May 1999 to March 2002. HITS was a Rowland circle spectrograph with a ruled, toroidal grating (3600 lines $/ \mathrm{mm})$ fed by an off-axis parabolic telescope (1/8 $\mathrm{m}$ focal length). The instrument operated as a limb imager with a field-of-view of $0.06^{\circ} \times 4.6^{\circ}$ and a fieldof-regard of $17^{\circ} \times 4.6^{\circ}$, covering the $100-750 \mathrm{~km}$ altitude range. It observed $110 \AA$ passbands of the $500-1800 \AA$ emissions from Earth's atmosphere and recorded spectra using a windowless, imaging microchannel plate detector.

[6] While the nominal operating mode of the HITS instrument was to scan the limb every $100 \mathrm{~s}$, it occasionally (2-3 days/month) operated in a "stare mode," where the viewing angle of the instrument was held fixed. The HITS data used in this study were obtained while HITS was observing in "stare mode," with tangent altitudes of 180-195 km, during a two day period in July 2001 . The data consist of spectra with $1.3 \AA$ resolution from the $1440-1550 \AA$ wavelength region, which includes $\mathrm{N}_{2} \mathrm{LBH}$ emissions from all of the a state vibrational levels. Since the sensitivity of HITS was low, individual 1-s samples of spectra with solar zenith angles less than $50^{\circ}$ are summed by latitude $\left(10^{\circ}\right.$ bins) to increase the signal-to-noise ratio (SNR) before fitting the observations to obtain temperatures. Each of these sums will be referred to as a "latitude spectrum." The number of 1-s spectra in these sums varies from 394 to 2366 and the SNR varies from 64 to 148 . While the individual 1-s spectra are binned only with respect to latitude, the spectra within each latitude bin come from multiple orbits, and therefore, different longitudes. Due to the Earth's rotation, the orbit-to-orbit change in longitude is $\sim 25.4^{\circ}$, but the longitude variation along the portion of each orbit used is only $\sim 2^{\circ}$. In order to account for longitude differences when fitting the observations, an airglow spectrum is modeled for each orbit contributing to a bin, using the average longitude of each orbit. The modeled spectra for each orbit are then averaged to give the final modeled spectrum that is fit to the observed spectrum for that bin. Further detail is provided in Section 3.

[7] In the observed spectra there is a large $(\sim 20 \%$ of the peak of the $(1,1)$ LBH band), wavelength-independent background contribution, which is attributed to the scattering of light within the HITS instrument. Due to the use of a ruled grating, the bright hydrogen Lyman-alpha (1216 $\AA$ ) and oxygen $1304 \AA$ emissions are significant sources of scattered light at all the wavelengths observed. The emissions in the observed passband also contribute to the background through scattering, but such contributions are orders of magnitude less than both the emissions themselves and the observed background. While the grating scatter contributions from the $1216 \AA$ and $1304 \AA$ emissions will not be completely wavelength independent, we believe the amount of variation over the wavelength range used in the analysis (1460 $1480 \AA$ ) will be insignificant due to the wavelength separation between the LBH bands being fit and the two (1216 and $1304 \AA$ ) dominant sources of the scattered light. To verify this we have examined nightglow observations from HITS. Since the $1216 \AA$ nightglow is orders of magnitude brighter than any other emission feature, the nightglow observations can be used to determine the wavelength dependence of $1216 \AA$ scattered light. Thousands of nightglow spectra were summed for each of the two days used in this study. The resulting spectra for both days appeared to be constant with respect to wavelength, and there are no identifiable grating ghosts. Linear fits to the nightglow data show that the change in background level is less than $1 \%$ over the $1460-1480 \AA$ spectral range. While this does not specifically address scattering from the $1304 \AA$ emission, we believe the analysis strongly supports the assumption of a uniform background when fitting the spectra from HITS.

[8] The passband of the HITS instrument was continuously selectable through rotation and translation of the grating. Due to mechanical irregularities, the same step position does not always return the mechanism to the same physical position, which results in a shift of the wavelength scale from that assumed. While the grating position was not changed during the collection of data used in the current analysis, the shift from the assumed wavelength scale must still be determined when fitting the spectra from HITS.

[9] In order to fit the HITS observations, it is necessary to know the tangent altitude associated with each measurement. Any error in the tangent altitudes will cause temperatures retrieved from the HITS data to be assigned to the wrong altitude. Unfortunately, due to a GPS failure early in the mission, ARGOS, and consequently HITS, was unable to accurately determine tangent altitudes. However, the analysis of HITS limb scans from July 28-30 [Eastes et al., 2011], which showed good agreement between HITS emission profiles and modeled profiles, suggests that the actual tangent altitudes were $16.9 \pm 2.4 \mathrm{~km}$ higher than indicated by the pointing data. This altitude shift is consistent with observations of a bright star (HD108073) on 29 July by both HITS and another instrument aboard ARGOS, the Low-Resolution Airglow and Aurora Spectrograph (LORAAS). For both instruments the discrepancy, 4-5 s or $\sim 0.34$ degrees, between the time the star was observed and the time expected from the pointing data is consistent with the discrepancy in tangent altitude noted by Eastes et al. [2011]. For the current analysis, a shift in tangent altitude equivalent to that calculated by Eastes et al. [2011] (16.9 \pm 2.4$)$ is assumed when fitting observed spectra to retrieve temperature. The tangent altitudes of the HITS observations used in this study vary with latitude from 180 to $195 \mathrm{~km}$ (including the shift mentioned above); however, the variation within each bin is only $\sim 2-4 \mathrm{~km}$. To account for the change in tangent altitude with latitude, the average tangent altitude of the observations contributing to each latitude bin is used when fitting that bin. Although the relationship between tangent altitude and temperature is nonlinear, the departure from linearity over a region of $2-4 \mathrm{~km}$ (at tangent altitudes of $180-195 \mathrm{~km}$ ) is small enough that using the average tangent altitude of each 


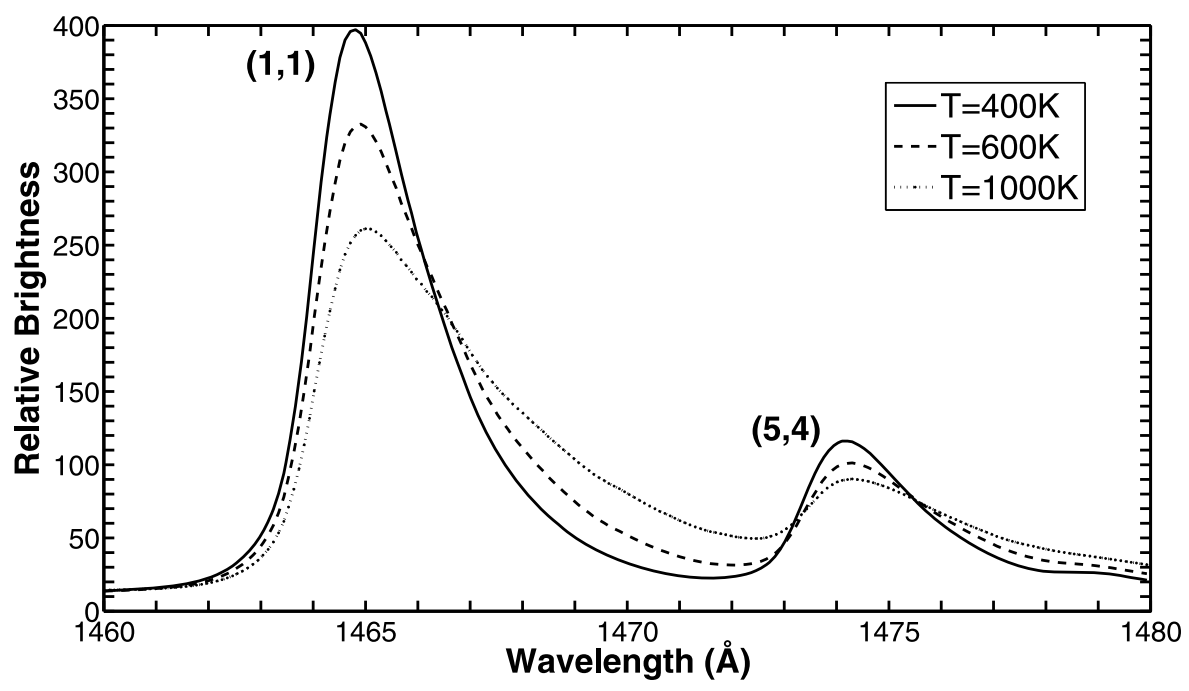

Figure 1. Broadening of the $(1,1)$ and $(5,4)$ emissions with increased rotational temperature enables remote sensing of rotational temperatures in the thermosphere.

bin is justified, since the impact on retrieved temperatures is less than $1 \mathrm{~K}$.

\section{Temperature Retrievals From HITS Data}

[10] At sufficient spectral resolution $(\sim 2 \AA$ for the LBH bands) the shape of molecular emission bands can be used to determine the temperature of the molecules producing the emission. An example of how the band shape depends on rotational temperature is shown in Figure 1. An increase in the rotational temperature broadens the band, increasing the emission from the longer wavelengths (due to an increase in the population of the higher rotational quantum states) relative to the shorter wavelengths. As discussed by Aksnes et al. [2006] the electron impact excitation of the $\mathrm{N}_{2}$ singlet states is not expected to significantly alter the rotational temperature, and the rotational and neutral gas temperatures in the Earth's thermosphere should be equal, given the collision and relaxation rates for the $\mathrm{N}_{2}$ molecules. This is consistent with the results of previous comparisons [e.g., Kurihara et al., 2003; Aksnes et al., 2006] between rotational temperatures and various versions of the Mass Spectrometer and Incoherent Scatter model atmosphere. In this paper, a more realistic modeling approach than the one described previously by Aksnes et al. [2006] is used to retrieve temperatures from HITS observations, and the retrieved temperatures are compared to temperatures from NRLMSISE-00 constrained to match densities derived from satellite drag. Using satellite drag data to constrain NRLMSISE-00 provides a check on temperatures calculated by NRLMSISE-00 when using only measured proxies for the solar and geomagnetic drivers.

[11] The rotational temperature information in the HITS $\mathrm{N}_{2}$ LBH dayglow observations can be extracted using inversion techniques that search for the best fit between modeled spectra and those observed. The observed spectrum is the sum of emissions from a range of altitudes (and consequently temperatures) along the line-of-sight (LOS). To fit this spectrum, the LBH emission from the 1460-1480 $\AA$ region, which includes the $(1,1)$ and $(5,4) \mathrm{LBH}$ bands, is modeled as the sum of the emissions from multiple altitudes $(\sim 115)$ along the LOS, each having a different temperature (Figure 2). The modeling incorporates the date, time, latitude, longitudes, tangent altitude, and geomagnetic conditions (Ap) appropriate for each latitude spectrum from HITS. The variation in brightness along the LOS is calculated using the Global Airglow (GLOW) [e.g., Solomon et al., 2001] and Intrasystem Cascade Excitation (ICE) [Eastes, 2000] models. The temperature (and neutral density) variation along the LOS is calculated using the NRLMSISE-00 model, and the solar proxies used by NRLMSISE-00 (daily and 81-day average F10.7) are used as fitting parameters. As F10.7 is varied, the thermospheric temperatures from NRLMSISE-00 change. This changes the rotational temperature of the $\mathrm{N}_{2}$ emissions and, consequently, the shape of the modeled spectrum.

[12] The nonlinear, least squares fitting of the modeled spectrum to that observed uses a modified LevenbergMarquardt scheme to perform Discrete Inverse Theory retrievals [Bevington, 1969; Menke, 1989; Meier and Picone, 1994]. With this method, the model is first calculated using initial values for the fitting parameters. The model is then compared to the observation, and the fitting parameters are iteratively adjusted until the fit converges. The fitting routine provides both the best fit parameters, as well as an estimate of the error on those parameters by calculation of the covariance matrix. Fitting a modeled spectrum to each latitude spectrum from HITS is done using a three-step process: 1) An initial fit of the 1440-1550 $\AA$ passband is done to obtain an estimate of the background; 2) The 1489-1499 $\AA$ region, which includes the atomic nitrogen emission at $1493 \AA$, is fit in order to determine any shift in the wavelength scale from that assumed; and 3) The 1460-1480 A region is fit to obtain the desired temperature information. A description of each step, including the model inputs and fitting parameters used, follows.

[13] Step 1. To determine an initial estimate of the background level in the data, an initial fit of the latitude spectrum is performed over the 1440-1550 $\AA$ passband using a single temperature. The fitting parameters used in this step are: scaling coefficients for the emission from each of the $\mathrm{v}=0$ 


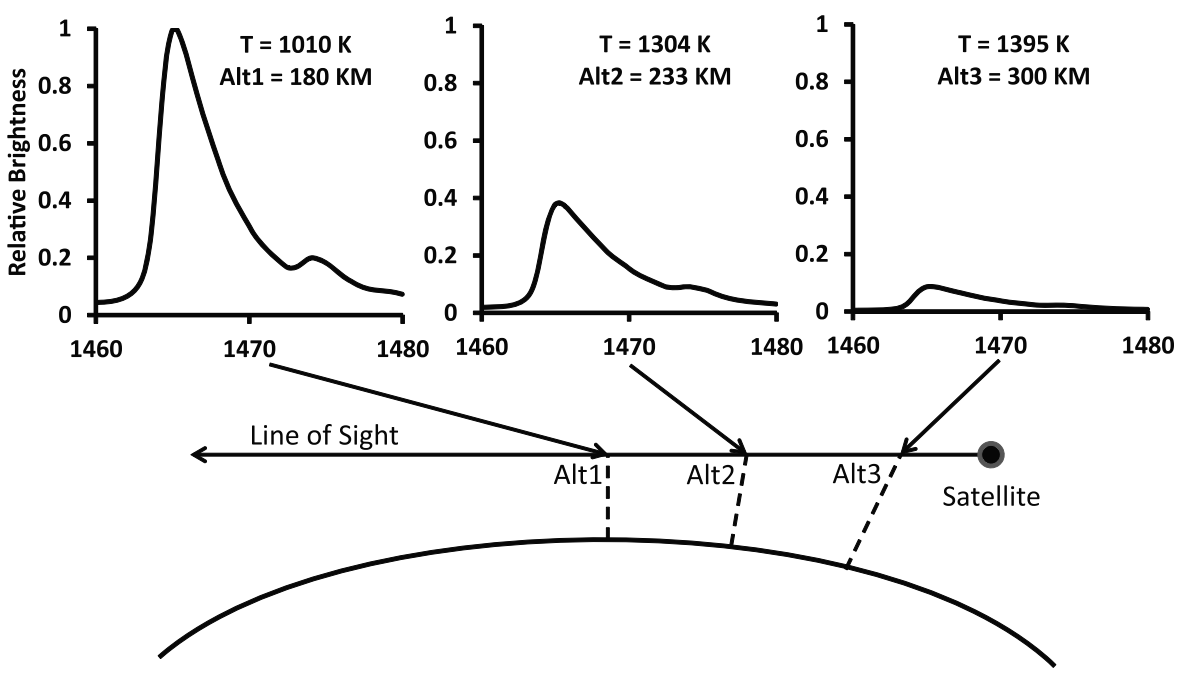

Figure 2. The LBH emissions observed by HITS are modeled as a superposition of spectra along the line of sight. Each point along the line is at a different altitude, and the calculated temperature and volume emission rate at each point is used to create a synthetic spectrum. Each spectrum is then attenuated by the effect of $\mathrm{O}_{2}$ photoabsorption and the individual spectra are summed to obtain the final model result. Spectra from the tangent altitude and two other points along the line of sight are shown.

through $\mathrm{v}=6 \mathrm{~N}_{2}$ vibrational levels, a scaling coefficient for the atomic nitrogen emission at $1493 \AA$, the $\mathrm{N}_{2}$ rotational temperature, and a uniform background. These parameters are used to model a synthetic spectrum that is compared to the observed spectrum. The values of the parameters are varied by the fitting routine until the fit converges. An example of this initial fit is shown in Figure 3. The dashed blue line is the uniform background level from the fit, the green line is the $\mathrm{N}_{2} \mathrm{LBH}$ contribution to the modeled spectrum (plus the background), and the red line, which is the final fit for this step, is the sum of the $\mathrm{N}, \mathrm{N}_{2}$, and background contributions.

[14] Step 2. Next, any wavelength shift in the observed spectrum is determined using a small wavelength region (1489-1499 §) around the atomic nitrogen doublet at $1493 \AA$. Since the wavelength of the atomic feature is not affected by the atmospheric temperature, any shift in its position is attributed to a shift in the wavelength scale. For this step, the background level determined in Step 1 is used as the initial guess for the background. The fitting parameters used are the same as in Step 1, except for the addition of a

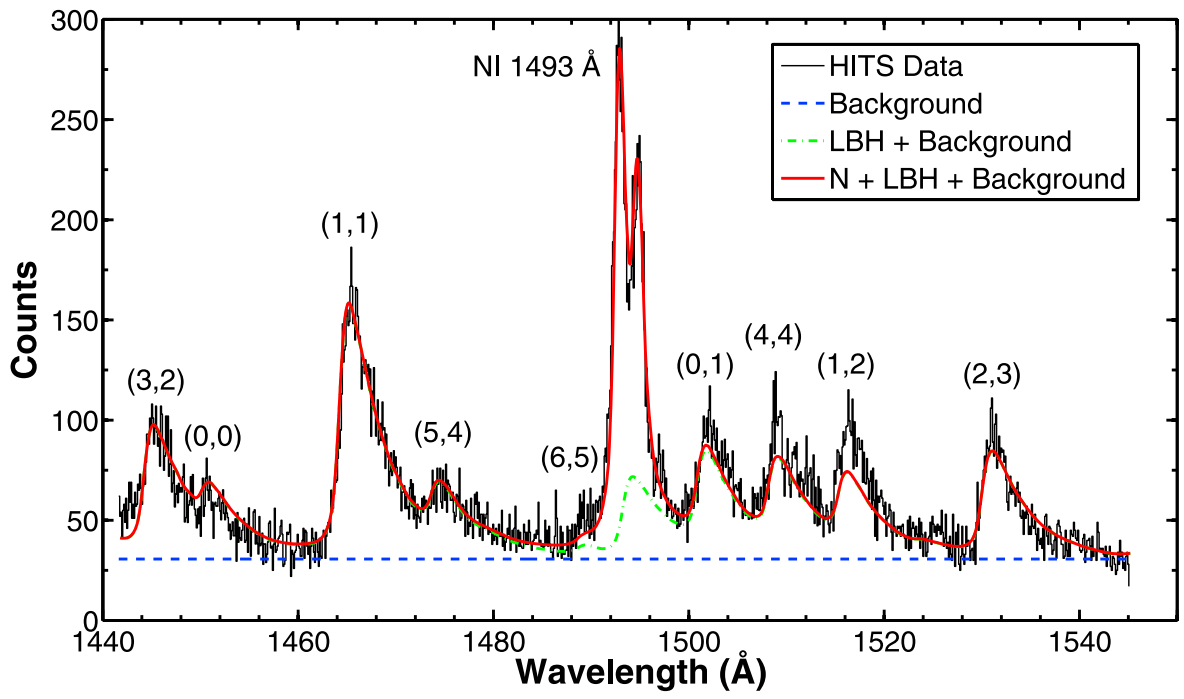

Figure 3. LBH spectrum in the 1440-1550 $\AA$ passband. The black line is the sum of spectra measured by HITS in the latitude bin near $66^{\circ}$ on 23 July 2001. The dashed blue line is the uniform background level determined by the fit described in Step 1, the green line is the $\mathrm{N}_{2} \mathrm{LBH}$ contribution to the modeled spectrum (plus the background), and the red line, which is the model fit for Step 1, is the sum of the $\mathrm{N}_{2} \mathrm{~N}_{2}$, and background contributions. The prominent bands are annotated with the vibrational states (initial, final). 


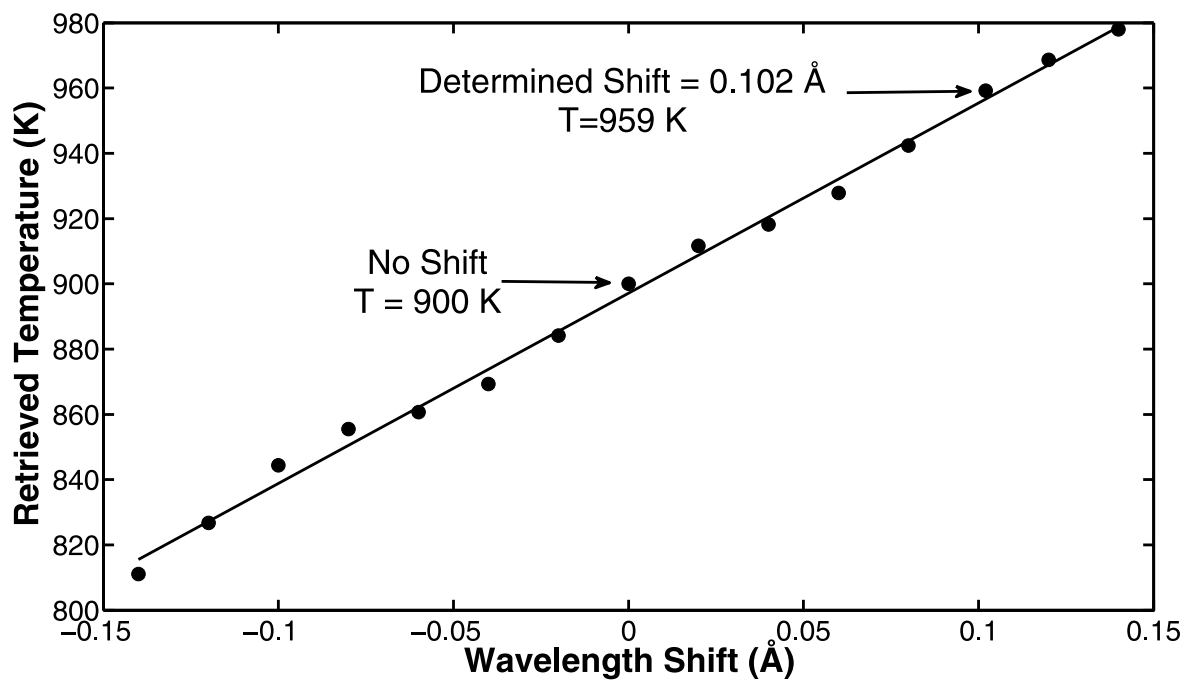

Figure 4. Effect of a shift in the assumed wavelength scale on the retrieved temperature for the $-10^{\circ}$ latitude bin on 23 July 2001. The relationship is linear, and similar results are seen for all latitude bins on both days. With no shift in the assumed wavelength scale, the retrieved temperature is $900 \mathrm{~K}$. Using the optimum shift determined in Step 2, the retrieved temperature is $959 \mathrm{~K}$.

parameter for the wavelength shift. Figure 4 shows the effect of a shift in the wavelength scale on the retrieved temperature (from Step 3) for the $-10^{\circ}$ latitude bin on July 23, 2001. Results are similar for all of the latitude bins on both days. As seen in the figure, the relationship between the shift and retrieved temperature is linear. With no shift in the assumed wavelength scale, the retrieved temperature is $900 \mathrm{~K}$. Using the optimum wavelength shift determined by the procedure described above, the retrieved temperature is $959 \mathrm{~K}$.

[15] Step 3. In the final step, the observed LBH emission from the 1460-1480 $\AA$ region is fit to obtain the atmospheric temperature. While the first two steps described above used only a single temperature to fit the observed spectrum, this final step incorporates the variation in temperature and brightness along the LOS into the fitting. The model inputs include the date, time, latitude, longitudes (one for each orbit contributing to the bin), tangent altitude (average value for the bin), and geomagnetic conditions (Ap) corresponding to each latitude spectrum from HITS, as well as any wavelength shift in the HITS data determined in Step 2 above. The parameters varied during the fitting are: scaling coefficients for the emission from the $\mathrm{v}=1$ and $\mathrm{v}=5 \mathrm{~N}_{2}$ vibrational levels, a uniform background, and F10.7 (the daily and 81-day average F10.7 values are set equal to each other during the fitting). While the background value determined in the initial fitting of the 1440-1550 $\AA$ region is used as an initial guess, the background is still varied when fitting the 1460-1480 $\AA$ region. As described below, the model is used to fit each latitude spectrum from HITS.

[16] First, the altitude of points along the LOS from the sensor through the tangent point of the observation is calculated at $30 \mathrm{~km}$ steps ( 115 steps) using the average tangent altitude for the latitude bin. This is done for each orbit that contributes to the bin, using the average longitude of each orbit, in order to account for the orbit-to-orbit longitude differences.
[17] Next, for each orbit that contributes, the temperatures and LBH volume emission rates are calculated at each step along the LOS for that orbit using the NRLMSISE-00, GLOW, and ICE models. To do this, the date, time, average latitude, average longitude, and Ap value corresponding to the orbit (and bin) are input into NRLMSISE-00, which is used to model the atmosphere (neutral densities and temperature). The solar proxies used by NRLMSISE-00 (daily and 81-day average F10.7) are fitting parameters, and are used to vary the temperature (and consequently the shape of the modeled spectrum). The atmosphere produced by NRLMSISE-00 is used by the GLOW model to calculate volume excitation rates for the a ${ }^{1} \Pi_{g}$ state. The ICE model, which calculates the effects of cascade on the LBH bands, then uses the volume excitation rates from GLOW to calculate the final LBH volume emission rates. These models are used to determine the variation of LBH brightness along the LOS, but not the total brightness itself. Instead, fitting parameters are used to adjust both the total LBH brightness and the relative brightness between the $\mathrm{v}=1$ and $\mathrm{v}=5$ vibrational levels.

[18] The calculated temperature (from NRLMSISE-00) and LBH volume emission rate at each step along the LOS are then used to create a synthetic spectrum for each step. The calculated spectrum from each step is attenuated by the $\mathrm{O}_{2}$ photoabsorption along the LOS to the sensor (HITS on ARGOS), multiplied by the normalized HITS instrument sensitivity (to account for variation of the sensitivity with wavelength), and convolved with the HITS instrument lineshape. The modeled spectra from each step along the LOS are summed to give the calculated spectrum for that LOS.

[19] The calculated spectra from each LOS (one for each orbit contributing to the bin) are then summed to give the modeled spectrum corresponding to the latitude bin. This modeled spectrum is compared to the observed latitude spectrum from HITS, and the process is iterated by varying the values of the fitting parameters until the fit converges. 


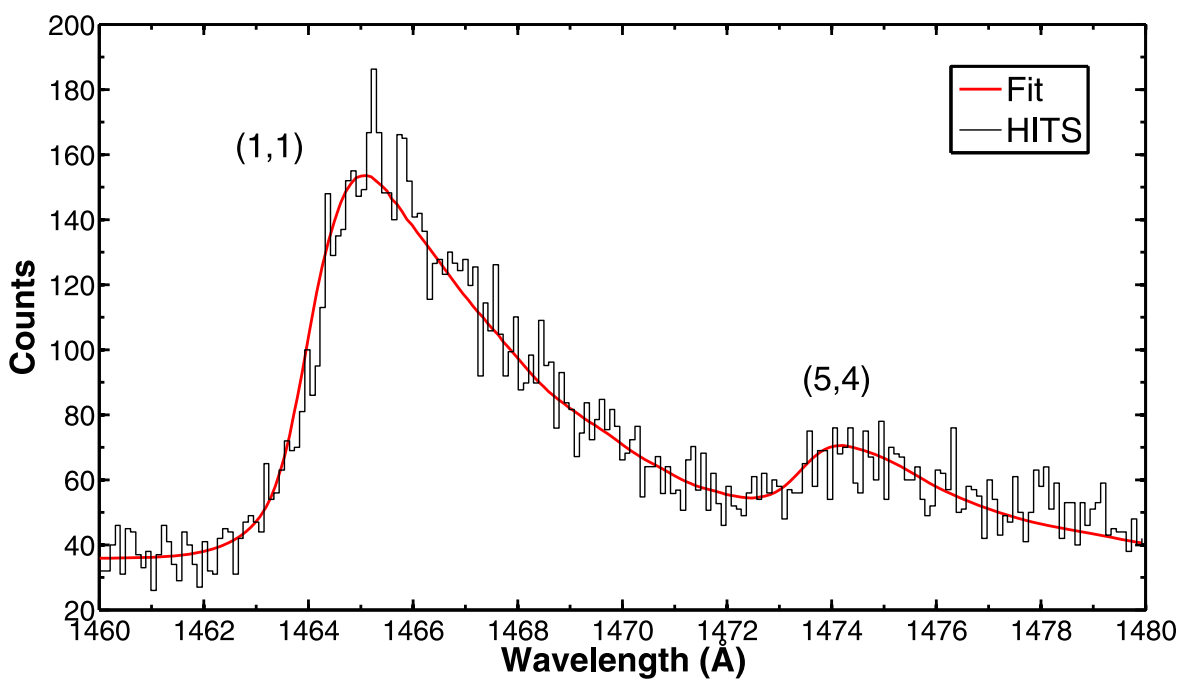

Figure 5. LBH spectrum in the $1460-1480 \AA$ region showing the $(1,1)$ and $(5,4)$ band peaks. The black line is the sum of spectra measured by HITS in the latitude bin near $66^{\circ}$ on 23 July 2001 . The red line is a modeled synthetic spectrum fit to the data using the process described in Step 3.

An example of the final model fit (red line) to a HITS spectrum (black line) is shown in Figure 5.

[20] The process described above is repeated for each latitude bin. The F10.7 values determined by the fitting process are then used with NRLMSISE-00 to obtain an atmospheric temperature corresponding to each latitude bin. This is done by using NRLMSISE-00 to calculate a temperature for each longitude (orbit) that contributes to the bin and then averaging. For comparisons of the results, we calculate the temperatures for each of the latitude bins at a reference altitude of $180 \mathrm{~km}$ instead of the actual tangent altitude $(180-195 \mathrm{~km})$ for each latitude bin.

[21] An uncertainty for the temperature is determined from the uncertainty on F10.7 that is provided by the fitting routine (calculated from the covariance matrix). This is done by using NRLMSISE-00 to calculate the temperatures again, using the (best fit) F10.7 value for the bin plus, or minus, its uncertainty, and once again averaging over the longitudes of the HITS observations.

\section{Satellite Drag Data}

[22] Satellite drag data are one of the most reliable sources of data for characterizing thermospheric density. The data used in this analysis are daily neutral density values calculated using drag data from 12 satellites (Table 1). The densities (one for each of the 12 satellites per day) were obtained from Frank Marcos (personal communication, 2008), and come from a database of high-accuracy, one-day resolution thermospheric neutral densities derived from tracking of 38 satellites [Marcos et al., 2006]. The densities were calculated using the method outlined by Bowman et al. [2004], and have estimated errors of less than 5\% [Marcos et al., 2006]. These densities are also a subset of the data used by Bowman et al. [2008] to update their empirical model of the neutral densities.

[23] The 12 satellites used in the present analysis had perigee altitudes of $405 \mathrm{~km}$ or less $(220-405 \mathrm{~km})$, and their perigees were almost uniformly distributed at latitudes below $70^{\circ}$ except for one satellite with a perigee near $80^{\circ}$. The orbits of the satellites are sufficiently elliptical to ensure that the drag, and consequently the calculated density, are spatially localized. The distribution of drag with respect to latitude was examined by Marcos et al. [2006]. The results of their study showed the majority of the drag is localized over a portion of the orbit near the point of closest approach. In addition, for a given perigee altitude, the drag becomes increasingly more localized as the apogee of the orbit increases. Using elliptical orbits therefore allows the designation of altitude, latitude, and local time to densities derived from satellite drag.

\section{Temperatures Calculated Using Satellite Drag Data}

[24] Recent analysis by Emmert et al. [2010] found that the average (total mass) densities and temperatures are both lower than expected (versus a solar proxy - F10.7 - driven

Table 1. Orbit Information for the 12 Satellites Used in the Drag Analysis $^{\mathrm{a}}$

\begin{tabular}{lccc}
\hline Satellite Number & Inclination $($ Deg) & Perigee $(\mathrm{km})$ & Apogee $(\mathrm{km})$ \\
\hline 22277 & 34.9 & 228 & 6888 \\
6073 & 52.1 & 231 & 5108 \\
4053 & 30.2 & 257 & 2580 \\
23853 & 82.9 & 293 & 1118 \\
8063 & 89.2 & 320 & 2378 \\
614 & 82.1 & 323 & 2107 \\
2389 & 81.4 & 359 & 2876 \\
2150 & 82.4 & 364 & 3738 \\
8133 & 25.3 & 380 & 1095 \\
4221 & 102.7 & 383 & 1855 \\
60 & 49.9 & 394 & 1069 \\
8744 & 82.9 & 408 & 1467 \\
\hline
\end{tabular}

${ }^{\mathrm{a}}$ Values given for the perigee and apogee are averages for 23-24 July 2001. 
model, NRLMSISE-00) during the recent solar minimum. They attributed mass density anomalies to a combination of lower-than-expected exospheric temperature and density reductions near the base $(\sim 120 \mathrm{~km}$, far below the $180 \mathrm{~km}$ tangent altitude of the HITS observations) of the diffusive portion of the thermosphere. Since most of the differences they found, (between NRLMSISE-00 and drag data), were not attributable to the F10.7 values, which have excellent long-term but poor short-term correlation with the solar (short-wavelength) irradiance forcing the atmosphere, some verification of the model result is desirable. Otherwise, the agreement between temperatures from the NRLMSISE-00 and the retrieved values could be fortunate coincidence, even if remote sensing observations from months of observations were used in the analysis. Their analysis also indicates that when the modeled densities between 200 and $400 \mathrm{~km}$ are constrained to match drag data, changes in composition at low altitudes $(\leq 120 \mathrm{~km})$ would result in $<16 \mathrm{~K}$ differences in the exospheric temperature.

[25] In order to calculate temperatures using the satellite drag data, the densities derived from satellite drag measurements are first used to constrain the NRLMSISE-00 model. The constrained model is then used to calculate temperature, averaged over the geographical positions covered by the HITS observations. The work of Marcos et al. [1998], whose derived densities (from satellite drag) are used in the current analysis, demonstrated for the first time that errors in predicted density could be reduced, by a factor of three globally, with a drag measurement from one satellite. Since the total density and temperature are interdependent in the NRLMSISE-00 model, we constrained NRLMSISE-00 with densities derived from drag measurements of 12 satellites to provide a check on the temperatures from NRLMSISE-00 when using only proxies for the solar and geomagnetic drivers.

[26] To constrain NRLMSISE-00, the daily and 81-day average F10.7 values are adjusted until the total density from the model agrees with the density values obtained from the drag measurements for each of the 12 satellites. The daily and average F10.7 values are assumed to be equal during this process, and the altitude, latitude and local time of the point of closest approach of the satellite are used as inputs for NRLMSISE-00. The F10.7 values determined from each of the satellites are then averaged to determine an F10.7 value for the day. For 23 and 24 July, the derived F10.7 values are $154.9 \pm 8.8$ and $148.2 \pm 8.2$ respectively. The uncertainties on the average F10.7 values are the standard deviation of the F10.7 values determined for each of the 12 satellites.

[27] The F10.7 values derived for each of the two days are then used with the NRLMSISE-00 model to calculate a neutral temperature corresponding to each of the latitude bins from HITS. This is done by first calculating the temperature at an altitude of $180 \mathrm{~km}$ for each orbit that contributes to the bin, using the average latitude of the bin, the average longitude of the orbit, and the derived F10.7 value for the day. The weighted average of those temperatures is the temperature expected from the model for that latitude bin. An estimated error for the temperature is determined by performing the NRLMSISE-00 calculation again using the derived F10.7 value plus, or minus, the error on that value. Since the geomagnetic activity level was relatively low $(\mathrm{Ap}=9.1$ and $\mathrm{Ap}=11.8$ for 23 and 24 July, respectively) during the observations, the assumption of hydrostatic equilibrium, that comes with the reliance on the NRLMSISE-00 model, is reasonable.

\section{Comparison and Results}

[28] A previous study of temperatures retrieved from HITS observations of the $\mathrm{N}_{2}$ LBH bands [Aksnes et al., 2006] found reasonable agreement with temperatures calculated using the NRLMSISE-00 and MSISE-90 models with measured values of the daily and average F10.7 solar parameters. However, the variation of temperature along the LOS of the HITS observations was not taken into account during the fitting done in that study. Instead, the observations were fit using a single temperature, which was then adjusted to account for temperature variation along the LOS, as detailed in Aksnes et al. [2006]. In the current study, the temperature variation along the LOS is used in modeling the $\mathrm{N}_{2}$ emissions during the fitting of the HITS observations. The temperatures retrieved from the HITS observations are compared to temperatures from NRLMSISE-00, both unconstrained and constrained to match the total densities derived from coincident satellite drag measurements.

[29] Figures 6 and 7 show temperatures obtained by inversion of HITS dayglow spectra (black squares) for the two days included in this study (23 and 24 July 2001). The values plotted (as black squares) in Figures 6 and 7 are the temperatures at an altitude of $180 \mathrm{~km}$ calculated with NRLMSISE-00, using the F10.7 value determined from fitting each HITS observation, as well as the latitude and longitude information corresponding to each latitude bin. These values are plotted as a function of the latitude of the observation. Neutral temperatures from the constrained NRLMSISE-00 model are also shown (red circles). These temperatures are also for an altitude of $180 \mathrm{~km}$, using the average F10.7 values derived from the drag data for each day, as well as the latitude and longitude information corresponding to each HITS latitude spectrum. The 1-sigma, statistical uncertainties for both the HITS and constrained NRLMSISE-00 temperatures are shown by the error bars on the data. For comparison, temperatures calculated using the (unconstrained) NRLMSISE00 model with measured geophysical parameters (Table 2) for the two days are also shown in Figures 6 and 7. In all three cases (HITS, constrained NRLMSISE-00, and unconstrained NRLMSISE-00), the temperatures shown represent temperatures at $180 \mathrm{~km}$ averaged over the longitudes covered by the HITS observations for each latitude bin.

[30] Good agreement between the temperatures from HITS and those from NRLMSISE-00 (both unconstrained and constrained with satellite drag data) is seen on both days. The absolute differences between the HITS and constrained NRLMSISE-00 temperatures on July 23 vary between $5.5 \mathrm{~K}$ and $73.7 \mathrm{~K}$, with a mean absolute difference of $21.7 \mathrm{~K}$. For July 24 , the absolute differences vary from $0.5 \mathrm{~K}$ to $47.3 \mathrm{~K}$, with a mean absolute difference of $20.3 \mathrm{~K}$. The temperatures from the constrained NRLMSISE-00 model are $\sim 10 \mathrm{~K}$ higher than temperatures from NRLMSISE-00 alone (unconstrained) on 23 July. On 24 July, the constrained NRLMSISE-00 temperatures are $\sim 7 \mathrm{~K}$ lower than the unconstrained NRLMSISE-00 temperatures.

[31] The 1-sigma statistical uncertainties for the HITS temperatures vary with the signal-to-noise ratio (SNR) of the HITS spectrum at each latitude, ranging from $\pm 22.8 \mathrm{~K}$ 


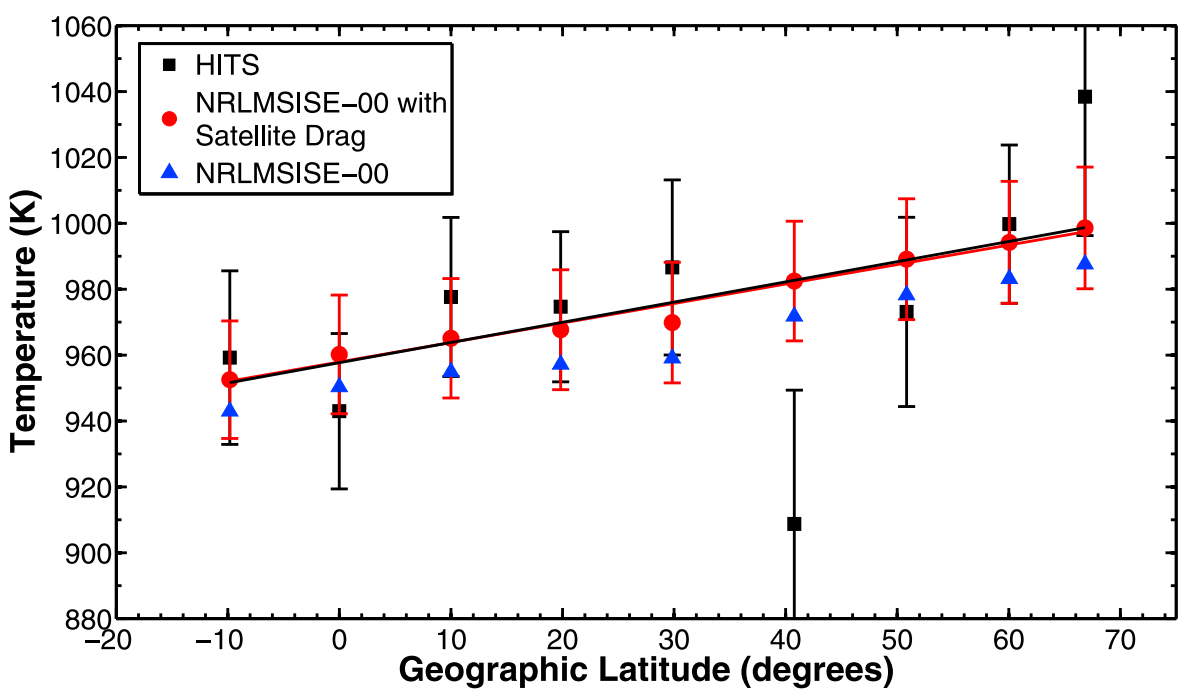

Figure 6. Neutral temperatures from 23 July 2001 obtained by inversion of high spectral resolution satellite observations of $\mathrm{N}_{2} \mathrm{LBH}$ emissions (squares), from NRLMSISE-00 constrained with densities derived from satellite drag data (circles), and from NRLMSISE-00 alone (triangles). The black and red lines are weighted least squares linear fits of the HITS and constrained NRLMSISE-00 temperatures, respectively.

$(\mathrm{SNR}=117)$ at $20^{\circ}$ latitude to $\pm 42.2 \mathrm{~K}(\mathrm{SNR}=64)$ at $40^{\circ}$ latitude on July 23 and from $\pm 17.2 \mathrm{~K}(\mathrm{SNR}=148)$ at $10^{\circ}$ latitude to $\pm 28.2 \mathrm{~K}(\mathrm{SNR}=89)$ at $40^{\circ}$ latitude on July 24 . The uncertainties for the constrained NRLMSISE-00 temperatures are approximately $\pm 19 \mathrm{~K}$ for July 23 and $\pm 18 \mathrm{~K}$ for July 24. For July 23, 8 out of 9 of the HITS and constrained NRLMSISE-00 temperatures agree within their statistical uncertainties, while for July 24,7 out of 9 temperatures are in statistical agreement.

[32] On both days, the HITS temperature at the $40^{\circ}$ latitude bin is significantly lower than the modeled temperature. We believe that this is due to chance. The SNR for the $40^{\circ}$ latitude bin is low on both days, as it also is for the highest latitude bin on 23 July, where a relatively high temperature was retrieved from the data. The SNR for the $40^{\circ}$ bin is low because the
HITS data were processed to avoid possible auroral contamination. When the spacecraft passed through the auroral oval, approximately $60^{\circ}-70^{\circ}$ latitude, HITS was observing near $40^{\circ}$ latitude. Many of the spectra in that latitude bin had significant deviations from the brightness expected in the dayglow and were not used in the analysis.

[33] Also plotted in Figures 6 and 7 are weighted-least squares linear fits to the HITS (black line) and constrained NRLMSISE-00 (red line) temperatures. These linear fits are not meant to be quantitative, but are intended to show that similar behavior with respect to latitude is seen in both sets of temperatures.

[34] As noted in Section 2, the observed HITS tangent altitudes were adjusted by $16.9 \mathrm{~km}$ to correct for an error in measured pointing data. Any error in this adjustment would

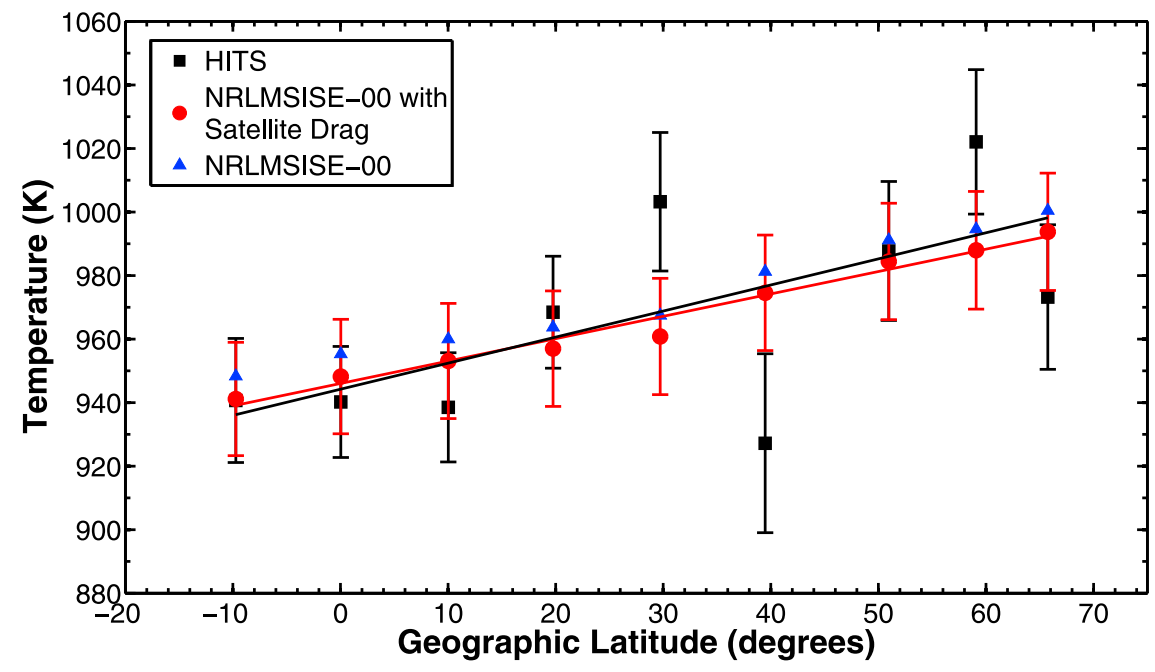

Figure 7. Same as Figure 6, but for 24 July 2001. 
Table 2. Geophysical Parameters Used When Calculating Temperatures With NRLMSISE-00 Alone ${ }^{\mathrm{a}}$

\begin{tabular}{lcc}
\hline Parameter & 23 July 2001 & 24 July 2001 \\
\hline F10.7 & 140.4 & 143.2 \\
F10.7 (81-day average) & 155.9 & 156 \\
Ap & 9.1 & 11.8 \\
\hline
\end{tabular}

${ }^{\mathrm{a}}$ Not constrained by densities derived from satellite drag.

result in all of the HITS temperatures being raised or lowered, depending on the sign of the error. The $\pm 2.4 \mathrm{~km}$ uncertainty on the $16.9 \mathrm{~km}$ value determined by Eastes et al. [2011] translates into an additional, systematic temperature uncertainty of approximately $\pm 6 \mathrm{~K}$ when fitting the HITS spectra.

\section{Conclusion}

[35] In this paper, the rotational structure of the LBH bands of $\mathrm{N}_{2}$ have been used to measure the neutral temperature in the lower thermosphere $(\sim 180 \mathrm{~km})$ by fitting modeled spectra to high resolution $(\sim 1.3 \AA)$ spectra measured by the HITS instrument aboard the ARGOS satellite on 23 and 24 July 2001 . The $(1,1)$ and $(5,4)$ LBH bands of $N_{2}$, which were observed at $\sim 1.3 \AA$ resolution, were used to determine the average rotational temperature as a function of latitude for each day. These rotational temperatures show good agreement, in both magnitude and latitudinal variation, with neutral temperatures from the NRLMSISE-00 empirical model in comparisons both with and without a constraint on the model (i.e., requiring the densities to match those from coincident, satellite drag data).

[36] The remotely sensed temperatures, from approximately $180 \mathrm{~km}$, and the neutral densities from satellite drag, from satellites between 200 and $400 \mathrm{~km}$, are both consistent with the modeled temperature, and corresponding density, profile for the lower thermosphere. Similar agreement was obtained on both days. Since both the data and model are longitudinally averaged (over the same latitudes and longitudes) this agreement indicates that the daily mean temperatures from the model are consistent with observations in the lower thermosphere during this geomagnetically quiet period. The agreement also suggests that observations of the LBH bands and their analysis are understood sufficiently for more extensive use in exploration of the lower thermosphere, a region which is sparsely measured but one which plays an important role in the behavior of the thermosphereionosphere system at higher altitudes.

[37] Acknowledgments. The work conducted at the University of Central Florida was supported by NASA grant NNG06GI94G and by NSF grant 0850396 . One of the authors (R. Eastes) wishes to thank Frank Marcos for providing the total thermospheric densities, from satellite drag measurements, used in the analysis and for his patience in educating the author about thermospheric densities.
[38] Robert Lysak thanks the reviewers for their assistance in evaluating this paper.

\section{References}

Aksnes, A., R. Eastes, S. Budzien, and K. Dymond (2006), Neutral temperatures in the lower thermosphere from $\mathrm{N}_{2}$ Lyman-Birge-Hopfield (LBH) band profiles, Geophys. Res. Lett., 33, L15103, doi:10.1029/ 2006GL026255.

Bevington, P. R. (1969), Data Reduction and Error Analysis for the Physical Sciences, McGraw-Hill, New York.

Bowman, B. R., F. A. Marcos, and J. J. Dendra (2004), A method for computing accurate daily atmospheric density values from satellite drag data, paper presented at 14th AAS/AIAA Flight Mechanics Meeting, Am. Astronaut. Soc., Maui, Hawaii.

Bowman, B. R., W. K. Tobiska, F. A. Marcos, C. Y. Huang, C. S. Lin, and W. J. Burke (2008), A New Empirical Thermospheric Density Model JB2008 Using New Solar and Geomagnetic Indices, Paper 2008-6438 $A m$, Inst. of Aeronaut. and Astronaut, New York.

Christensen, A. B., et al. (2003), Initial observations with the Global Ultraviolet Imager (GUVI) in the NASA TIMED satellite mission, J. Geophys. Res., 108(A12), 1451, doi:10.1029/2003JA009918.

Dymond, K. F., K. D. Wolfram, S. A. Budzien, C. B. Fortna, and R. P. McCoy (1999), High-resolution Ionospheric and Thermospheric Spectrograph (HITS) on the Advanced Research and Global Observing Satellite (ARGOS): Quick look results, Proc. SPIE, 3818, 137, doi:10.1117 12.364149 .

Eastes, R. W. (2000), Modeling the $\mathrm{N}_{2}$ Lyman-Birge-Hopfield band in the dayglow: Including radiative and collisional cascading between the singlet states, J. Geophys. Res., 105, 18,557-18,573, doi:10.1029/ 1999JA000378.

Eastes, R. W., D. J. Murray, A. Aksnes, S. A. Budzien, R. E. Daniell, and A. Krywonos (2011), Modeled and observed $\mathrm{N}_{2}$ Lyman-Birge-Hopfield band emissions: A Comparison, J. Geophys. Res., 116, A12308, doi:10.1029/2010JA016417.

Emmert, J. T., J. L. Lean, and J. M. Picone (2010), Record-low thermospheric density during the 2008 solar minimum, Geophys. Res. Lett., 37, L12102, doi:10.1029/2010GL043671.

Kurihara, J., K.-I. Oyama, K. Suzuki, and N. Iwagami (2003), Vibrationalrotational temperature measurement of $\mathrm{N}_{2}$ in the lower thermosphere by the rocket experiment, Adv. Space Res., 32(5), 725-729, doi:10.1016/ S0273-1177(03)00407-1.

Marcos, F. A., M. Kendra, J. Griffin, J. Bass, J. Liu, and D. Larson (1998), Precision low earth orbit determination using atmospheric density calibration, J. Astronaut. Sci., 46, 395.

Marcos, F. A., B. R. Bowman, and R. E. Sheehan (2006), Accuracy of Earth's thermospheric neutral density models, paper presented at AIAA AAS Astrodynamics Specialist Conference, Am. Astronaut. Soc., Keystone, Colo.

Meier, R. R. (1991), Ultraviolet spectroscopy and remote sensing of the upper atmosphere, Space Sci. Rev., 58(1), 1-185, doi:10.1007/ BF01206000

Meier, R. R., and J. M. Picone (1994), Retrieval of absolute thermospheric concentrations from the far UV dayglow: An application of discrete inverse theory, J. Geophys. Res., 99(A4), 6307-6320, doi:10.1029/ 93JA02775.

Menke, W. (1989), Geophysical Data Analysis: Discrete Inverse Theory, Academic, San Diego, Calif.

Picone, J. M., A. E. Hedin, D. P. Drob, and A. C. Aikin (2002), NRLMSISE-00 empirical model of the atmosphere: Statistical comparisons and scientific issues, J. Geophys. Res., 107(A10), 1468, doi:10.1029/ 2002JA009430.

Solomon, S. C., S. Baily, and T. Woods (2001), Effect of solar soft X-rays on the lower ionosphere, Geophys. Res. Lett., 28(11), 2149-2152, doi:10.1029/2001GL012866

Strickland, D. J., J. S. Evans, and L. J. Paxton (1995), Satellite remote sensing of thermospheric O/N2 and solar EUV, 1. Theory, J. Geophys. Res., 100, 12,217-12,226, doi:10.1029/95JA00574. 